\title{
Can "words" be processed as integrated units?
}

\author{
WAYNE P. SILVERMAN \\ New York State Institute for Basic Research in Mental Retardation, Staten Island, New York 10314
}

\begin{abstract}
Three experiments examined the information processing of letters embedded within one-syllable words and similar unpronounceable sequences. A speeded discrimination task was used to detect processing differences between words and nonwords in a situation where both the identity and position of critical display information was known to subjects before stimulus presentation. Results indicated that word pairs differing by two letters were more quickly discriminated than two words differing in a single letter, while nonword pairs differing in two letters were discriminated no faster than two nonwords differing in a single letter. A further comparison showed a performance advantage for words over nonwords in a condensation task that forced a scan of stimulus letters for correct responding. These results suggest that familiarity affects information processing at a perceptual level, and are incompatible with theories suggesting that familiarity effects are due to inferential factors following letter feature analysis.
\end{abstract}

Recent investigations of letter perception have found that performance increases as letter sequences more closely approximate the regularities of acceptable English spelling patterns (e.g., Barron \& Pittinger, 1974). The "word superiority effect" (WSE) has been demonstrated reliably in paradigms using tachistoscope recognition (e.g., Reicher, 1969) and "same-different" reaction time paradigm (e.g., Barron \& Pittinger, 1974), and current theories of word perception have focused on processing differences between letters embedded in words and random letter sequences.

Two general classes of theories have been proposed to account for the WSE. The first can be labeled as the perceptual class, and suggests that the perceptual units by which letter strings are analyzed differ between words and nonwords. These theories all suggest that unpronounceable sequences are processed letter by letter. The perceptual units for words differ in the various formulations. Smith (1971) suggested that words were processed as wholes. Gibson (1965) suggested that the perceptual unit was a "spelling pattern," defined as "a cluster of graphemes in a given environment which has an invariant pronounciation according to the rules of English" (p. 1071). In addition, Spoehr and Smith (1973) have proposed that the perceptual unit of word analysis is the "vocalic center group," a unit approximately syllable sized. These theories all have one element in common. The WSE is caused by a

I thank Gail Bruder, Lew Hill, Edward Smith, and anonymous consulting editors for their comments on earlier drafts of this paper. Portions of these data were presented at the Forty-sixth Annual Meeting of the Eastern Psychological Association, New York, 1975, and the Sixteenth Annual Meeting of the Psychonomic Society, Denver, 1975. The address of the New York Institute for Basic Research in Mental Retardation is 1050 Forest Hill Road, Staten Island, New York 10314. differentiation between words and nonwords in a "unitization" process, and processing differences are due to the size of the respective perceptual units. Performance differences are observed because the units are processed sequentially, while features within the units are processed in a functionally parallel fashion. The total time necessary to process an entire word is therefore reduced relative to the time required to process a nonword containing the same number of letters (with the obvious exception of one-letter words).

The second class of theories will be referred to herein as inferential or postperceptual. These theories suggest that the perceptual analysis of letter features is the same for both words and nonwords, and performance difference between the two sequence types are caused by processing mechanisms operating after the analysis of visual letter features (e.g., Estes, 1975a, b). Support for this type of theory comes from several experiments based on Reicher's (1969) tachistoscope recognition procedure. Massaro (1973) and Thompson and Massaro (1973) found that the WSE vanishes when subjects are shown response alternatives before stimulus presentation, and argue that this manipulation is a more adequate control of redundancy factors that may be operating when response alternatives are unknown during stimulus presentation [as in the postexposure cue condition of Reicher (1969)]. Further, Bjork and Estes (1973) showed that when the visual features of the letter responses were known before stimulus presentation the WSE also vanished. These results suggest that the WSE arises because of uncertainty and the advantage of words over nonwords due to inherent redundancy of transitional letter probabilities.

To reconcile the conflicting results from studies finding a WSE (e.g., Reicher, 1969) and those failing 
to find the effect (e.g., Massaro, 1973), Estes $(1975 \mathrm{a}, \mathrm{b})$ proposed a hierarchical theory of letter (and word) perception. The flow of information within this model goes from the input to visual feature analyzers, then to a letter identification stage, and finally to a multiletter unit identification stage (Estes, 1975a, p. 20). The output of earlier stages serves as the input to later stages, and context affects all processing levels. The WSE will not be found if response decisions are made solely on the basis of visual feature analysis, and will be found when the analysis must proceed to higher levels (e.g., letter identification).

Determinants of the level of analysis are context and task demands. Estes (1975a) suggests that when subjects are aware of the critical features for which to scan, the processing system is preset for detection of these features and no WSE should be found. In contrast, when visual features are unknown before stimulus presentation, this preactivation cannot occur. Subjects will then be required to identify letters as stimulus components, as this represents the efficient method of coding the stimulus for memory and response requirements. When the task demands this level of analysis, a WSE will be found due to the greater redundancy inherent in letter sequences forming words. Estes suggests that the WSE arises chiefly from a reduction in positional uncertainty.

The present investigation was designed to test between these two broad classes of theories, the critical difference being the location in the processing scheme where linguistic context has its effect. The essential conceptual point of the perceptual theories (e.g., Gibson, 1965; Spoehr \& Smith, 1975) is that words are processed in multiletter units while nonwords are analyzed letter by letter. Estes $(1975 a, b)$ suggests that the visual letter feature analysis stage is identical for both words and nonwords. To choose between these two alternatives, a task is needed that controls for the possible effects of nonperceptual processing differences between words and nonwords. Estes' (1975a, pp. 21-22) discussion suggests that four functions of linguistic context must be controlled or eliminated from consideration. These potential word-nonword differences include: (a) guessing strategy differences based on partial information; (b) response threshold or criterion differences; (c) positional uncertainty differences after partial information analysis; and (d) setting factor differences, such as instructions.

All of these criteria seem to be met if: (a) subjects know before stimulus presentation where critical item information will occur in the display and what the critical visual features are; and (b) task performance will be sensitive to the size of the unit of perceptual analysis. Selection of an appropriate task obviously requires a preliminary definition of "per- ceptual unit." Garner (1974) has suggested that some stimuli are processed as a single perceptual unit, and called these integral stimuli. In contrast, separable stimuli are analyzed by their component units (or features). These concepts will serve to define the notion of perceptual unit for the present discussion. If the Spoehr and Smith (1973) theory is correct, isolated syllables should behave like integral stimuli. If the Estes (1975a, b) model is correct, both familiar and unfamiliar letter sequences should behave like separable stimuli. (Although there are several logical problems raised by the proposal that any visually presented material, separated spatially, is processed as an integral stimulus, this framework does serve to define empirical tasks that differentiate between the perceptual and inferential theories of the WSE. Discussion of related theoretical problems will be deferred to later sections of this paper.)

\section{EXPERIMENT I}

Garner (1974) pointed out several empirically defining properties of integral and separable stimuli. Biederman and Checkosky (1970) used a two-choice reaction time (RT) task that could make the distinction between the two stimulus types. Their subjects' task was to discriminate between two possible stimulus alternatives. The alternatives differed on either one or two relevant dimensions and RTs to discriminate pairings differing on two dimensions were shorter than pairings differing on only a single dimension. This improvement in performance, termed a redundancy gain, should occur when integral stimuli are proeessed. When separable stimuli are processed, Garner's (1974) framework predicts that the RTs to discriminate between pairs differing on two dimensions should be no faster than the easier unidimensional discrimination, yet no slower than the more difficult unidimensional discrimination. ${ }^{1}$

Biederman and Checkosky (1970) detailed the theoretical difficulties involved in comparing a bidimensional discrimination (redundant condition) to a unidimensional discrimination. The most serious potential confound is the problem of controlling for individual subject differences in the ability to process the component dimensions. To illustrate, if the two dimensions are size and shape, it is conceivable that half of the subjects find size the easier discrimination while the remainder are faster at discriminating shape. The outcome of this situation must be an averaging of faster and slower RTs for each unidimensional discrimination. However, in the redundant condition, every subject can process the preferred dimension. Therefore, the mean RT for the redundant discrimination could be lower than either of the unidimensional discriminations, even if subjects are processing separable stimuli. 
To avoid this problem Biederman and Checkosky (1970) compared the redundant condition of each subject to the faster of that subject's unidimensional discriminations, thus controlling for individual preferences. This control, however, raised another problem. When each subject responds to two unidimensional conditions and one redundant condition, the faster unidimensional condition has the greatest chance probability of being the fastest discrimination. As the redundancy gain, if present, is likely to be of small magnitude, this sampling advantage favoring the faster unidimensional discrimination has a reasonable chance of obscuring the gain. Biederman and Checkosky (1970) controlled for this factor by presenting subjects with two redundant conditions, equalizing the a priori probabilities that the fastest condition will be the faster of either the two unidimensional or two redundant conditions.

The Biederman and Checkosky (1970) method was modified for use with letter-sequence stimuli. A letter was arbitrarily defined as a feature, and subjects were required to discriminate between two sequences differing in either one or two letters. If the Spoehr and Smith (1973) type model is correct, results should show: (a) no difference between words and nonwords when discriminations are based on a singleletter difference, replicating the results of Massaro (1973) and Thompson and Massaro (1973), where subjects knew the locus of critical information before test trials; (b) no redundancy gain for nonword discriminations differing in two letters; and (c) a redundancy gain for word discriminations differing on two letters. In contrast, if the Estes (1975a b) type theory is correct, the feature analysis of the individual letters should be the same for both words and nonwords; there should be no interaction between the various discrimination conditions and wordness categories.

\section{Method}

Design. The experiment was a 2 by 4 design. There were two levels of Wordness (Words and Nonwords) and four different Discriminations (two based on one letter and two based on two letters). Discrimination was a within-subjects variable, while Wordness was a between-subjects variable. The stimuli and discriminations for the Word stimuli are shown in Figure 1.

Subjects and apparatus. Thirty-two adults, staff members of the Institute, participated in the experiment. All were native readers of English. Stimuli were presented on a Scientific Prototype two-field tachistoscope. Subjects responded by pushing one of two response keys with either their right or left hands, and their reaction times and responses were recorded to the nearest millisecond by a Hewlett-Packard (5223L-5050A) counterprinter combination.

Stimuli. There were two stimulus conditions, words and nonwords, within which the specific letter discriminations had to be carefully selected. If one letter pairing was highly discriminable (e.g., $X$ and $O$ ) while the other pairing was difficult to discriminate (e.g., $Q$ and $O$ ), the possible redundancy gain would be minimized, as its magnitude is determined by the relative increase in stimulus discriminability with the addition of the

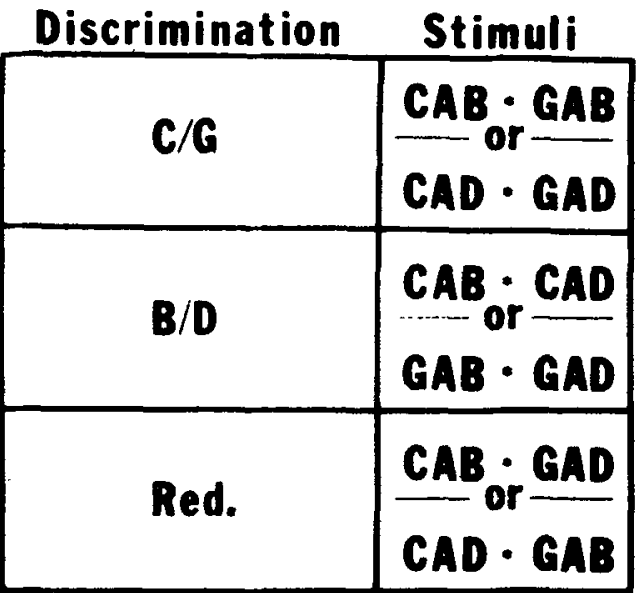

Figure 1. The word stimuli in Experiment I. Each subject viewed one pair from each of the $C / G$ and $B / D$ discriminations and both redundant discriminations.

redundant information. Therefore, the greater the divergence in discriminability between pairings, the smaller the potential redundancy gain. Also, even if the two one-letter discriminations are equal in discriminability, each pair must be relatively difficult to differentiate in order to avoid a ceiling effect. The expected redundancy gain is small in magnitude, and will not be measurable if subjects can maximize their performance in the one-letter discrimination conditions. [Biederman and Checkosky (1970) found an average redundancy gain of only $18 \mathrm{msec}$ in their experiment, p. 489].

These considerations make stimulus selection extremely difficult. The source of all word stimuli was, therefore, Webster's Unabridged Dictionary (1966). No further consideration of word familiarity was made in stimulus selection.

The specific word stimuli were CAB, CAD, GAB, and GAD. The nonword stimuli, CJB, CJD, GJB, and GJD, were formed by substituting a randomly picked consonant for the vowel of the words. ${ }^{2}$ Stimuli were constructed on approximately $13 \times 25 \mathrm{~cm}$ white index cards using Chartpak transfer letters (Franklin Gothic Condensed, $18 \mathrm{pt}$ ). Stimuli were viewed from a distance of $81.3 \mathrm{~cm}$ and subtended horizontal visual angles of $47 \mathrm{~min}$.

Procedure. The subjects were seated in front of the tachistoscope, located in a dimly lit room. Half the subjects were randomly assigned to the word condition and the remainder to the nonword condition. The subjects were then instructed as to the exact nature of the task, with speed and accuracy of response emphasized. On each trial, the subjects were required to signal which of two possible stimuli was presented. Each stimulus was assigned to a response key and the response assignments and stimulus possibilities were held constant within each block of trials. Each subject was presented with four blocks-of experimental trials. One block required subjects to discriminate on the basis of only the first letter with the third held constant, while a second block required subjects to discriminate on the basis of the third letter with the first held constant. The remaining two blocks allowed discriminations based on either the first or third letters. These various conditions are outlined in Figure 1.

Each block contained 24 trials, 12 each of the two possible stimuli. The order of the last 20 trials within each block was random, the only constraint being that no more than five consecutive trials required the same response. The first four trials in each block were counted as practice. The order of block presentation was counterbalanced in a Latin square design. For each one-letter discrimination block, there are two possible stimulus pairings, as shown in Figure 1. Each possibility was used for one-half the subjects. In addition, the response assignments for the two stimulus pairs were counterbalanced. 
Table 1

Mean Reaction Times (Milliseconds) and Error Rates (\%) for the Various Discriminations in Experiment 1

\begin{tabular}{lccccc}
\hline & \multicolumn{2}{c}{ RT } & & \multicolumn{2}{c}{$\%$ error } \\
\cline { 2 - 3 } \multicolumn{1}{c}{ Discrimination } & Word & $\begin{array}{c}\text { Non- } \\
\text { word }\end{array}$ & & Word & $\begin{array}{c}\text { Non- } \\
\text { word }\end{array}$ \\
\hline C/G & 535 & 523 & & 4.7 & 3.1 \\
B/D & 508 & 491 & 4.1 & 2.5 \\
CB-GD & 490 & 514 & 5.0 & 3.1 \\
CD-GB & 500 & 504 & 3.8 & 1.9 \\
Faster one-letter of each subject & $509^{*}$ & $497^{*}$ & & \\
Faster two-letter of each subject & $492^{*}$ & $505^{*}$ & & \\
\hline
\end{tabular}

Note-These values are based on the individual subject's mean RTs.

At the beginning of each block of trials, the subjects were shown the two stimuli that were to be discriminated, along with their response assignments. Before the first block of experimental trials began, the subjects were given 30 practice trials on stimuli consisting of $\mathrm{Xs}$ and Os. The procedure for the practice block was identical to the experimental trials. Each trial consisted of: (a) a verbal ready signal; (b) the onset of a $1-\mathrm{sec}$ warning stimulus (a rectangle surrounding the area where the stimulus was to appear) which also functioned as a fixation field; (c) the onset of the stimulus (either the $X$ or the $O$ for practice trials and one of the two possible letter sequences for experimental trials) for $30 \mathrm{msec}$; (d) the offset of the stimulus followed by a dark field; (e) the depression of one of the two response keys, stopping the electronic counter, and recording the subject's RT (to the nearest millisecond) and response; and ( $f$ ) the positioning of the next stimulus. There was no feedback given during the intertrial interval, which was approximately $3 \mathrm{sec}$. The entire experimental session lasted approximately $24 \mathrm{~min}$.

\section{Results}

The results are given in Table 1. Except where noted, the $\overline{\mathrm{RT}} \mathrm{s}$ represent the means of the individual subjects' median RTs for that condition calculated on the basis of only correct trials. The data were analyzed using a two-way analysis of variance (Wordness by Discrimination). There was no main effect of Wordness, but the main effect for Discrimination was significant, $F(3,90)=7.27, p<.01$, and was due solely to the slower RT of the C/G discrimination condition relative to the others, $F(1,90)=$ $22.0, \mathrm{p}<.01$.

The interaction also reached significance, $F(3,90)=$ $3.23, \mathrm{p}<.05$. Inspection of Table 1 shows that the interaction seemed to be due to changes in the relationship of the one-letter and two-letter discrimination for words and nonwords. This contrast was significant, $F(1,90)=7.71, p<.01$, and no other orthogonal component approached significance. Also, analysis using the Studentized range procedure showed that words and nonwords did not differ on any specific discrimination.

To test the redundancy gains, the mean RTs were calculated and, for each subject, the faster one-letter and two-letter discriminations were compared. Eleven of the 16 subjects viewing the word conditions showed redundancy gains, four showed a redundancy loss, and one subject showed equal RTs for the faster one- and two-letter discriminations. In the nonword condition, seven subjects showed redundancy gains and nine subjects showed redundancy losses. Analysis of variance of these data showed that there was no overall redundancy gain or overall Wordness effect, both $F s(1,30)<1.0$. The interaction was significant, $F(1,30)=6.04, p<.05$. Separate analysis of word data showed a significant redundancy gain, $F(1,15)=$ $5.10, p<.05$. Similar analysis of the nonword data showed no difference between the faster one- and two-letter discrimination, $F(1,15)=1.38, p>.25$. Although it seemed unlikely, differences between the various word discriminations may have been due to familiarity effects, as CAD and GAD are uncommon words. It is possible that some of the word pairs required, subjectively, word-nonword discriminations, and these were based on different information processing. A check was made to insure that this factor had no effect. Within the $B / D$ and the $C / G$ discrimination conditions, a comparison was made between the two stimulus pair alternatives. (For example, in the word condition, the $\mathrm{C} / \mathrm{G}$ comparison was between subjects viewing CAD-GAD and those viewing CAB-GAB). None of these comparisons reached significance for either words or nonwords.

The redundancy gain in the word condition may be explainable by reference to other, confounded factors. As discussed by Biederman and Checkosky (1970), such factors should be ruled out before finalizing conclusions. One possibility is systematic transfer differences across trial blocks presented first and second for both the two-letter and one-letter discriminations. This comparison showed that transfer favored the one-letter discriminations slightly. The mean drop in RT for the second unidimensional block was 19 msec, while the second redundant condition showed only a 9-msec performance improvement. This difference was not significant.

An additional factor that effects the magnitude of the redundancy gain is the overlap of the processing distributions. It is crucial that the two redundant processing distributions do not have a significantly -greater overlap than the two unidimensional processing distributions [see Biederman and Checkosky (1970) for a detailed theoretical discussion of this issue]. Although the average RT difference between the two one-letter discriminations of the word condition was slightly greater than the difference between the redundant conditions ( 27 and $10 \mathrm{msec}$, respectively), this difference was not significant, $t(15)=1.27, p>.2$.

The error data was analyzed in a fashion similar to the RT data. The overall error rate was $3.5 \%$. There were no statistically significant differences in error rates across conditions. Error rates for the various conditions are given in Table 1.

\section{Discussion}

The results of the first experiment support the 
hypothesis that letters within words and nonwords are processed differently. Because subjects knew in advance where to locate critical information, there was no Wordness effect when stimuli differed in a single position, replicating previous results (e.g., Thompson \& Massaro, 1973). However, the obtained Wordness by Discrimination interaction is suggestive of different perceptual mechanisms.

The strongest evidence of word-nonword processing differences is the interaction in the redundancy gain analysis. The data suggest that nonwords are separable stimuli, and are processed letter by letter, as the RTs in redundant conditions are no faster than the faster one-letter discrimination and no slower than the slower one-letter discrimination. Words, in contrast, seem to be integral stimuli, as a significant redundancy gain was present.

The interaction between words and nonwords cannot easily be explained by reference to differential letter scanning strategies. The $\mathrm{C} / \mathrm{G}$ discrimination, being both the slowest and leftmost comparison, would tend to be processed first more often for words than for nonwords if there was a scanning order difference. Such a strategy predicts results opposite in direction to those obtained.

The results of the first experiment provide support for the perceptual theories of the WSE and are difficult for the Estes $(1975 \mathrm{a}, \mathrm{b})$ model to accommodate. In reference to the Estes framework, the current task exemplifies the type of situation where no wordnonword differences should be found, as both critical visual features and their locations within the display were known to subjects before stimulus presentation. Further, as the same discriminations were required for many trials within the same block, familiarity effects, were minimized.

The most surprising aspect of the results was the redundancy gain for word stimuli. It may be possible that this result does not actually indicate that visually presented words are integral stimuli, as it is still possible that the redundancy gain for word stimuli was due to a sampling artifact. Because the $B / D$ discrimination was faster than the $C / G$ discrimination, subjects could have performed on the basis of the easier discrimination for both two-letter discriminations and only a single one-letter discrimination. Selecting the faster two-letter discrimination could, therefore, result in an apparent redundancy gain. This sampling problem cannot explain the wordnonword interaction, but it seems useful to eliminate it as a possible explanation of the redundancy gain for words. To accomplish this, a second experiment, equalizing the two one-letter discriminations, was designed.

\section{EXPERIMENT II}

The second experiment was an exact duplication of the word condition in the first experiment, with only minor procedural changes. As results from the first experiment failed to give any hint of a redundancy gain for nonword sequences, this condition was dropped. There were 16 subjects, and each experimental block of trials was lengthened to 34 trials. ${ }^{3}$ The first 10 trials in each block were considered practice. Further, half the subjects viewed the stimuli in a forward order and the remainder viewed them in reverse order to reduce any chance sequencing effects. All other procedural details were identical to the word condition in Experiment I.

The stimuli for this experiment were BIB, BID, DIB, and DID. The method of stimulus construction was the same as in the first experiment. These stimuli were selected so that subjects always chose between $B$ and $D$, therefore equalizing the difficulty of the two one-letter discriminations.

\section{Results}

The results are given in Table 2 . The means were again based on the individual subject's median RTs for correct trials only. The overall data were analyzed using a Subjects by Discrimination analysis of variance. There was a significant Discrimination effect, $\mathrm{F}(3,45)=9.90, \mathrm{p}<.01$. Follow-up comparisons showed that the effect was due to the difference between the DIB-BID discrimination and the other conditions, $F(1,45)=31.12, p<.01$. The other three conditions did not differ significantly.

A second comparison between conditions involved the mean RTs of the faster of the two one-letter and two-letter comparisons of each subject. Eleven of the 16 subjects were faster for the two-letter discrimination, two subjects' mean RTs were equal for these two conditions, and the remaining three subjects were slightly faster for the one-letter discrimination. A sign test showed that this performance advantage for the two-letter discrimination was significant, $\mathrm{p}<.03$ (one-tailed). The redundancy gain was significant in magnitude, $\mathrm{t}(15)=2.79, \mathrm{p}<.01$ (one-tailed).

Comparisons of the alternative stimulus pairings in the two one-letter discriminations showed no significant differences, and there was no difference between subjects viewing the stimuli in the forward or reverse orders. Again, to test for possible transfer and task learning effects, performance differences

Table 2

Mean Reaction Times (Milliseconds) and Error Rates (\%) for the Various Discriminations in Experiment II

\begin{tabular}{llc}
\hline \multicolumn{1}{c}{ Discrimination } & RT & \% error \\
\hline First letter & 445 & 4.4 \\
Third letter & 435 & 4.2 \\
DID-BIB & 426 & 2.9 \\
DIB-BID & 475 & 5.2 \\
Faster one-letter of each subject & $436^{*}$ & 3.4 \\
Faster two-letter of each subject & $426^{*}$ & 2.3 \\
\hline
\end{tabular}

Note-These values are based on individual subject's mean RTs. 
between the trial block presented first and the block presented second for both the one-letter and twoletter discriminations were compared. There was a slight transfer advantage favoring the one-letter discriminations over the two-letter discriminations. (The average performance gain for the second oneletter condition was $4.1 \mathrm{msec}$, while the second twoletter condition was slower than the first by $2.8 \mathrm{msec}$.) This difference was not significant, $t(15)=0.31$, $p>.5$.

Error rates for the various conditions are given in Table 2. The overall error rate was $4.2 \%$. There were no significant differences in error rates between the various conditions.

\section{Discussion}

The results of the second experiment reinforce the impressions from the first experiment. The conclusion seems to be that words are integral stimuli. The redundancy gain found in the second experiment cannot be accounted for by sampling artifacts resulting from discriminability differences between the two one-letter discriminations. If anything, a similar sampling artifact operated against the redundancy gain; as the DIB-BID discrimination proved to be most difficult.

The finding that the BID-DIB discrimination was slower than the others is, on face value, quite surprising. As it happened, evidence of this result was detected after testing the first three subjects, and all following subjects were interviewed after the experiment in order to learn how they accomplished the task. Subjects reported processing the "whole word" on some trials and a "single letter" on others, consistent with processing flexibility for words suggested by Aderman and Smith (1971). Subjects also reported that response assignments were made by assigning $B$ to one response and $D$ to the other response. For all possible stimulus pairings, this strategy works nicely, except for the BID-DIB discrimination. Here both the first and third letter can be either B or D and a situation for potential interference is encountered. This confusion was spontaneously reported by several subjects, and seemed to be the basis for the poor performance on the BIDDIB discrimination. However, this explanation raises some theoretical problems. Garner (1974) emphasized that integral stimuli must be processed as wholes, and analysis of the individual features is not a processing option. Therefore, some discussion of alternative theoretical explanations seems warranted, and will be presented after additional data are described.

\section{EXPERIMENT III}

It is possible that something inherent in the earlier discrimination tasks artificially differentiates between words and nonwords. This notion is contradicted by the specificity of the difference between words and nonwords in the first experiment, but when significance was found in the first two experiments, the effects were not terribly strong. Therefore, a third experiment was run to expand the results, strengthen conclusions, and seek an additional task demonstrating word-nonword differences.

Some differences between performance on words and nonwords seemed to depend on whether the task demanded that the whole stimulus be processed or whether subjects could perform by processing information from only a single letter position (e.g., Thompson \& Massaro, 1973). When only a single letter need be processed for successful task performance, there is no reason to expect to find a WSE, as such tasks may not differentiate between the size of the respective word-nonword perceptual units. Experiments where the position of critical information is uncertain demand that the whole stimulus be processed, and performance should increase as perceptual unit size increases. It seems that the WSE quite frequently is found in these designs (e.g., Eichelman, 1970), although there are instances where this is not the case (e.g.; Bjork \& Estes, 1973). Therefore, a discrimination task was selected that demanded the processing of information from more than one letter position before correct response selection. A condensation task (Fitts \& Biederman, 1965), where both the first and third letters of a stimulus must be identified is such a task. Furthermore, Gottwald and Garner (1975) demonstrated that performance in a condensation task was better when subjects viewed integral stimuli compared to their performance when viewing separable stimuli.. A clear advantage favoring words over nonwords in a condensation task, where the critical features and their display locations are still given to subjects before stimulus presentation, would strengthen previous arguments that perceptual analysis of familiar and unfamiliar material differs.

A redundant condition was also included in the design of Experiment III in order to further verify the existence of a redundancy gain for words. In order to reduce the number of discriminations subjects were required to make, the second redundant condition was omitted. This lowers the a priori probability of finding a redundancy gain, so positive evidence of its existence would add considerable strength to the argument that words were integral stimuli.

\section{Method}

Design. A 2 (word-nonword) by 4 (Discrimination) by Subjects design was used. All subjects received both words and nonwords. The four discriminations were: (a) first letter only, (b) third letter only, (c) first or third letter (redundant), and (d) first and third letter (condensation).

Subjects and apparatus. Sixteen adults, again members of the 
Institute staff, served as subjects. The apparatus was the same as in the previous two experiments.

Stimuli. The stimuli were four letter sequences. For the word condition, they were RARE, RAPE, PARE, and PAPE. To form the nonword stimuli, three raters selected consonants most similar in structure to the vowels of the word stimuli, and these consonants were substituted for the vowels to form unpronounceable nonwords. The stimuli were RXRF, RXPF, PXRF, and PXPF. Stimuli were typed on index cards identical to those used in the previous experiments by an IBM Selectric typewriter with an Orator alphabet. Stimulus letters were all capitalized, with a space typed between each letter. Stimuli subtended horizontal visual angles of $1^{\circ} 4^{\prime}$ when viewed by subjects.

Procedure. The procedure for this experiment was similar to that of the two previous studies. Subjects participated for 2 consecutive days, half receiving the word condition on the first day, with the remainder viewing the word condition on the second day. Within each block of trials, there were 52 stimuli, with the first 12 counted as practice. The order of the trials within the word condition was randomly determined, as before, but the nonword stimuli were ordered so as to require the same response sequence as in the word condition. Subjects received the nonword condition in the reverse order of the word condition, one-half receiving the words in the forward order and the remainder viewing the words in a reverse order. In this manner, any artifact of stimulus sequencing was counterbalanced. The construction of stimuli. in the one-letter discrimination conditions was analogous to that of the previous experiments. The redundant condition required subjects to discriminate between $P_{-} P_{-}$and $R_{-} R_{-}$. The condensation task required subjects to select one response if either $P_{-} P_{-}$or $R_{-} R_{\text {Las }}$ wresented, and the alternative response if either $\mathbf{P}_{-} \mathbf{R}-$ or $\mathbf{R}_{-} \mathbf{P}_{-}$was presented. For the condensation task, the probability of occurrence of each of the four possible stimuli was 0.25. Again, Discrimination block order was counterbalanced in a Latin square design, but each subject received the same block order for both words and nonwords.

\section{Results}

The results of the third experiment are given in Table 3. The RTs were first analyzed using a 2 by 2 by 4 (Wordness by Wordness Order by Discrimination) analysis of variance. Wordness and Discrimination were within-subject variables, while Wordness Order was a between-subjects variable. The analysis was done on the median RTs of the subjects for only correct responses. The only significant effect was for Discriminations, $F(3,42)=94.07, p<.01$, due to the slowness of the condensation task relative to the other discriminations, $F(1,42)=277.0$, $\mathrm{p}<.01$. Although the overall Wordness by Discrimination interaction was not significant, the predicted difference between the two condensation conditions was significant, $\mathrm{t}(15)=2.951, \mathrm{p}<.01$.

Evidence of a redundancy gain was present for 11 subjects in the word condition and nine subjects in the nonword and condition. Of the 11 subjects showing the gain in the word condition, 6 also showed a gain for nonwords. In neither condition was there a significant overall redundancy gain.

Again, as in Experiments I and II, data from the two one-letter conditions were analyzed to see if there were any effects due to the two different stimulus pair alternatives. No such differences were found.

The error data are given in Table 3, and analysis of variance, similar to that used for the RT data, showed a significant Discrimination effect, $F(3,42)=$ $20.22, p<.01$. This effect was due solely to the higher error rates for the condensation task relative to the other conditions, $F(1,42)=59.04, p<.01$. No other factor reached significance. The overall error rate was $6.4 \%$.

\section{Discussion}

The performance difference between the words and nonwords in the condensation task provides additional support for the notion that words are integral stimuli and nonwords are separable stimuli. Although critical item identity and location were known before stimulus presentation, and the specific letter discriminations were identical for both words and nonwords, a clear word advantage was found. This finding is incompatible with the Estes $(1975 \mathrm{a}, \mathrm{b})$ type account of familiarity effects on letter processing efficiency. The effect cannot be due to artifacts of stimulus sequencing, as the required response order was identical for both words and nonwords.

An alternative explanation of this result might suggest that subjects had greater trouble remembering which stimulus went with what response for the nonwords. However, the stimulus assignments were quite straightforward; if the first and third letters were the same, one response was appropriate; if they were different, the other response was correct. Subjects were made aware of this rule before performing in the condensation task, so memory processes seem minimized.

The failure to find a significant redundancy gain for the word condition is not important. The inclusion of only a single redundant condition provides an obvious explanation for its absence. Experiment III was designed to examine processing in the condensation task, and the probability of finding a redundancy gain for words was minimized intentionally. Although its presence would have strongly supported the notion that words were integral stimuli, its

Table 3

Mean Reaction Times (Milliseconds) and Error Rates (\%) for the Various Discriminations in Experiment III

\begin{tabular}{lllll}
\hline & \multicolumn{2}{c}{ RT } & & \multicolumn{2}{c}{$\%$ error } \\
\cline { 2 - 3 } \cline { 4 - 4 } Discrimination & Word & Nonword & Word $\quad$ Nonword \\
\hline
\end{tabular}

Overall Data

\begin{tabular}{lrcrr} 
First letter & 503 & 507 & 5.3 & 5.2 \\
Third letter & 530 & 531 & 3.8 & 5.2 \\
Redundant & 500 & 500 & 4.4 & 2.8 \\
Condensation & 693 & 721 & 13.3 & 11.8 \\
& \multicolumn{4}{c}{ First Condition of Subjects } \\
& 500 & 513 & 6.6 & 3.1 \\
First letter & 505 & 547 & 4.7 & 5.9 \\
Third letter & 486 & 516 & 4.4 & 2.5 \\
Redundant & 686 & 751 & 11.9 & 9.7 \\
Condensation & &
\end{tabular}


absence does not necessarily contradict this notion.

\section{GENERAL DISCUSSION}

These three studies, taken together, strongly imply that visually presented words are integral stimuli. If all the data from the word conditions are considered, 33 subject sessions showed a redundancy gain, 2 were exactly equal, and 11 showed an advantage favoring the one-letter discrimination. The chance probability of an outcome showing a difference of this magnitude favoring the two-letter discriminations is 0.0006 (sign test). On the other hand, for the nonword stimuli (presented only in Experiments I and III), a total of 16 out of 32 subjects showed evidence of a possible redundancy gain. This result obviously does not differ from chance; letters forming nonwords are separable stimuli. Taken together with the superior performance of words in the condensation task, these results support the broad class of perceptual theories of the WSE (e.g., Spoehr \& Smith, 1973) and contradict the inferential theories (e.g., Estes, 1975a). The prime considerations in this conclusion are: (a) the results, showing specific and predicted word-nonword differences; and (b) the procedure, which allowed subjects advance knowledge of what visual features to scan for and where in the display to focus attention.

Proponents of the inferential theories can argue that the sampling in this group of experiments was small, and therefore the results cannot readily be generalized. However, Clark (1973) has pointed out the value of experiments designed to test single cases. The results of Experiment I, supported by Experiments II and III, seem sufficiently incompatible with the Estes (1975a) theory, and represent in themselves strong counterarguments. Admittedly, these results cannot readily support a single, specific theory within the perceptual class (e.g., Smith, 1971), but support for this general theoretical family seems strengthened by process of elimination.

There are problems with the identification of visually presented words as integral stimuli and nonwords as separable stimuli. (The reader is reminded that this model was used only to suggest tasks that could empirically distinguish between perceptual and inferential theories of the WSE.) There are several reasons why the model fails. First, Garner (1974) has pointed out that features separated spatially are processed as separable stimulus components. This logical problem can be overcome if it is assumed that the visual input of the present experiments is recoded into phonemic representations and then discriminated. [Garner (1974) suggests that syllables presented auditorially may very well be integral stimuli.] Indeed, this assumption is made formally in the Spoehr and Smith (1975) theory, where a trans- lation of visual to phonemic information occurs after unitization but before perceptual analysis is completed. It is, however, not obvious why this recoding is necessary. The discrimination can certainly be performed on the basis of only the visual information, and Posner and Mitchell (1967) demonstrated that the recoding of visual into phonemic information entails a substantial loss in performance efficiency. Further, the present tasks epitomize the situation where responses should be based on the visual letter features (Estes, 1975a, b).

The second aspect of the present results that contradict the notion that words are integral stimuli comes from the finding in Experiment II that the BID-DIB discrimination was more difficult than the others. If subjects were accurate in their subjective reports, they processed the component letters. Garner (1974) stated that this should not be possible when integral stimuli are discriminated.

An alternative model, suggesting that both visually presented words and nonwords are separable stimuli, avoids these problems. One possible model suggests that letters within words are processed by a selfterminating, parallel, independent channel system or serially, letter by letter, depending on task demands (e.g., Aderman \& Smith, 1971). In contrast, nonwords would have to be processed serially. This model predicts the redundancy gains for words, the absence of redundancy gains for nonwords, and the condensation task advantage favoring words.

This hypothesis also has its faults. Previous studies of information processing of random letter arrays (nonwords) have supported a parallel, independentchannel model of letter perception (e.g., Colegate \& Eriksen, 1972; Eriksen \& Lappin, (1967). These results seem in direct conflict with the present data. The contradiction may be resolved by reference to Garner's (1974) distinction between state and process performance limitations. State limitations cause lowering of performance by decreasing stimulus energy and, consequently, decreasing the probability that critical stimulus information is input into the system. In contrast, process-limited performance is depressed because the discriminability between the various stimuli is decreased. Examination of the procedures and stimuli of the Colegate and Eriksen (1972) study, for example, showed that the letter stimuli were highly discriminable and performance in their task, tachistoscopic recognition, was state limited. In contrast, the present procedure had no appreciable state limitations (the dark poststimulus field allowed clear stimulus perception, and the low overall error rate supports this notion) and definite process limitations (the letter discriminations were selected to assure difficulty). This could explain why past evidence of parallel processing for nonword letter displays was not replicated in the present 
experiments. It seems reasonable to suggest that state limitations affect stimulus input processes while process limitations affect later stimulus analysis. As different processing stages may be affected by state and process variables, there is no reason to suspect that manipulation of one factor will affect results in a fashion similar to the manipulation of the other factor. In fact, Garner (1974) outlines the independent nature of the two classes of variables along with some differential effects on performance.

To come full circle, there is a final problem with the suggestion of parallel vs. serial processing differences between letters forming words and nonwords as the explanation for the WSE. Garner (1974) stated that, given only process limitations, no redundancy gains will be found unless subjects discriminate between integral stimuli. So it is clear that a finalized theoretical model, internally consistent and compatible with all relevant data is still elusive. The importance of the present results is their incompatibility with inferential theories of the WSE (e.g., Estes, 1975a).

The data presented here are compatible in concept with the Gibson (1965) and Spoehr and Smith (1973, 1975) formulations. Although the present investigation was not designed specifically to discriminate between these two theories, results are compatible only with the theoretical details of the Spoehr and Smith (1973) model. The two formulations basically differ in the proposed size of the perceptual "units" into which words are parsed for processing. Gibson (1965) suggested that the unit, termed a spelling pattern, is a "cluster of graphemes in a given environment which has an invarient pronounciation according to the rules of English" (p. 1071). In contrast, Spoehr and Smith (1973) suggested that the unit, now termed a "vocalic center group," was approximately syllable size. As the present results showed redundancy gains across spelling pattern boundaries, yet within the boundaries of vocalic center groups, the Spoehr and Smith formulation seems superior in its theoretical detail. However, it is still premature to now accept the syllable or vocalic center group as the "unit" in word perception. The present data cannot discriminate between the Spoehr and Smith (1973) theory and an alternative that proposes the entire word at the perceptual unit (e.g., Smith, 1971).

Whatever specific theory of word perception is eventually proven to be correct, the data presented here are strong support for the notion that words can function as integrated units, defined as integral stimuli or separable stimuli within which information has been integrated by processing mechanisms (Garner, 1974). Because the tasks employed allowed subjects to perform by processing only a single letter when viewing nonwords, the increase in processing efficiency due to familiarity had to be underestimated. When all letters must be processed to at least some degree, as in everyday reading behavior, the increased size of the perceptual unit should yield great performance gains.

\section{REFERENCES}

Aderman, D.. \& Smith, E. E. Expectancy as a determinant of functional units in perceptual recognition. Cognitive Psychology, 1971, 2, 117-129.

BARON, J., \& Thurston, I. An analysis of the word-superiority effect. Cognitive Psychology, 1973, 4, 207-228.

Barron, R., \& Pittinger, J. The effect of orthographic structure and lexical meaning on "same-different" judgements. Quarterly Journal of Experimental Psychology, 1974, 26, 566-581.

Biederman, I., \& Checkosky, S. Processing redundant information. Journal of Experimental Psychology, 1970, 83, 486-490.

BJORK, E., \& Estes, W. Letter identification in relation to lingu istic context and masking conditions. Memory \& Cognition, 1973, 1, 217-223.

Clark, H. The language-as-fixed-effect fallacy: A critique of language statistics in psychological research. Journal of Verbal Learning and Verbal Behavior, 1973, 12, 335.339.

Colegate, R., \& Erixsen, C. Form of redundancy as a determinant of tachistoscopic word recognition. Perception \& Psychophysics, 1972, 12, 477-481.

ERIKSEN, C., \& LAPPIN, J. Independence in the perception of simultaneously presented forms at brief durations. Journal of Experimental Psychology, 1967, 73, 468-472.

EichelmaN, W. Familiarity effects in the simultaneous matching task. Joumal of Experimental Psychology, 1970, 86. 275-282.

Estes, W. K. Memory, perception, and decision in letter identification. In R. Solso (Ed.) Information processing and cognition: The Loyola symposium. New Jersey: Lawrence Erlbaum. 1975. (a)

Estes, W. K. The locus of inferential and perceptual processes in letter identification. Journal of Experimental Psychology: General, 1975, 104, 122-145. (b)

FrtTs, P., \& Brederuan, I. S.R. compatibility and information reduction. Journal of Experimental Psychology, 1965, 69, 408-412.

GARNER. W. R. The processing of information and structure. Maryland: Lawrence Erlbaum, 1974.

Girson. E. Learning to read. Science, 1965, 148, 1066-1972.

GotTWALD, R., \& Garner, W. R. Filtering and condensation tasks with integral and separable dimensions. Perception \& Psychophysics, 1975, 18, 26-28.

Massaro, D. Perception of letters, words, and nonwords. Journal of Experimental Psychology, 1973, 100, 349-353.

Posner. M.. \& Mitchell. R. Chronometric analysis of classification. Psychological Review, 1967, 74, 392-409.

REIChER, G. Perceptual recognition as a function of meaningfulness of stimulus material. Journal of Experimental Psychology, 1969, 81, 275-280.

SMrrH, F. Understanding reading. New York: Holt, Rinehart. \& Winston, 1971.

Spoehr, K. T., \& Sмiтh, E. E. The role of syllables in perceptual learning. Cognitive Psychology. 1973, 5, 71-89.

SPOEHR. K. T., \& SMITH, E. E. The role of orthographic and phonotactic rules in perceiving letter patterns. Journal of Experi mental Psychology: Human Perception and Performance, 1975. 1. 21-34.

Thompson, M., \& Massaro, D. Visual information and redundancy in reading. Journal of Experimental Psychology, 1973, 98. 49-54. 
Webster's third new international dictionary of the English language unabridged. Springtield. Massachusetts: Merriam. 1966.

\section{NOTES}

1. Garner (1974) discusses paradigms in which, if stimulus information was difficult to detect (state limitations), separable dimensions can show redundancy gains. Such a situation can and was avoided in the present paradigm, as the stimuli were clearly visible.

2. In this manner, unpronounceable nonwords were guaranteed. This precaution was taken because Baron and Thurston (1973) showed that pronounceable nonwords could be processed like words and I wanted to maximize the chances for finding wordnonword differences.

3. Some of these subjects were also run in Experiments $I$ and III, as the availability of subjects was limited. Subject selection from the available pool was completely random for each experiment, as was the assignment of subjects to specific conditions within each experiment.

(Received for publication December 15, 1975; revision accepted June $21,1976$. ) 